\title{
Review of: "Poor nutritional status, risk of sarcopenia and nutrition related complaints are prevalent in COVID-19 patients during and after hospital admission"
}

Bilinc Dogruoz Karatekin

Potential competing interests: The author(s) declared that no potential competing interests exist.

Nutritional status and sarcopenia risks of COVID-19 patients at ICU and nursing ward discharge and after 1 month were evaluated. The selection and classification of the patient sample and the evaluation after discharge added value to the study. In future studies, sarcopenia should be evaluated with valid scales and tests, not the risk of sarcopenia. 